ISSN: 2523-2886

DOI: https://doi.org/10.17162/rmi.v3i2.1156

\title{
Relaciones directamente proporcionales entre la salud y el espacio: Un enfoque de salud preventiva desde una óptica arquitectónica
}

\author{
Magdalena Carrasco Díaz ${ }^{1}$ y Josué Pinedo Chávez ${ }^{2}$ \\ Universidad Peruana Unión, Perú ${ }^{12}$
}

Recibido: 15 de enero de 2018

Aceptado: 21 de julio de 2018

\section{Resumen}

El objetivo de este artículo es comprender el impacto del espacio en el bienestar de la persona, considerando la salud desde una perspectiva integral, a fin de proyectar espacios saludables en el que la arquitectura participe como instrumento de curación. Para ello, se estudian teorías sobre patologías urbanas, salud urbana, salud integral y la relación de los componentes arquitectónicos con el individuo. Se comprenderá finalmente tres aspectos esenciales: el espacio tiene un impacto directo en la salud de la persona; así también, los componentes de la arquitectura (luz, color, sonido, aromas, naturaleza, materiales) influyen en el estado físico, psicológico y espiritual del ser humano; por último, la proyección de espacios para la salud, deberá contemplar una visión holística de la persona, de no ser así, se estaría fragmentando al ser humano y dejando a un lado aspectos que conforman parte esencial de su salud.

Palabras clave: salud urbana, espacios saludables, salud integral, A-D-E-L-A-N-T-E

\section{Directly Proportional Relationships between Health and Space: A Preventive Health Approach from an Architectural View}

\begin{abstract}
The aim of this article is to understand the impact of space on the well-being of the person, considering health from an integral perspective, to project healthy spaces in which architecture participates as a means of healing. For this, theories about urban pathologies, urban health, integral health and the relationship of the architectural components with the individual are studied. Three essential aspects will be understood: space has a direct impact on the health of the person; likewise, the components of architecture (light, color, sound, smell, nature, materials) influence the physical, psychological and spiritual state of the human being. Accordingly, the projection of spaces for health should contemplate a holistic view of the person; if not, it will be fragmenting the human being and leaving aside aspects that make up an essential part of their health.
\end{abstract}

Keywords: urban health, healthy spaces, integral health, A-D-E-L-A-N-T-E

\footnotetext{
${ }^{1}$ Correspondencia con el autor

E-mail: magdalenacarrasco@upeu.edu.pe

${ }^{2}$ Correspondencia con el autor

E-mail: josuepinedo@upeu.edu.pe
} 


\section{Introducción}

Actualmente, un promedio de 38 millones de personas muere al año producto de las enfermedades no transmisibles (ENT); entre ellas, las principales son: enfermedades cardiovasculares (17.5 mll.), cáncer (8.2 mll.), enfermedades respiratorias (4 mll.) y la diabetes (1.5 mll.; Organización Mundial de la Salud, 2018). Las ENT afectan a diferentes edades y dependen directamente del estilo de vida de cada individuo. Este estilo de vida generalmente está condicionado por el entorno, pues el ser humano es influido constantemente por el ambiente que le rodea, ya que hombre y medio ambiente forman una unidad perceptiva (Ittelson, 1978) y no entidades separadas. Por ello, es importante estudiar teóricamente los aspectos fisiológicos, psicológicos y espirituales de la persona y cómo el espacio influye en ellos. El objetivo de esto es proponer ambientes que transmitan reacciones favorables en el cuerpo, mente y espíritu, asegurando de esta manera la salud integral del usuario.

Patologías urbanas

Las patologías urbanas se van desarrollando debido a la forma en la que hoy crecen las ciudades (INEI, 2015), de acuerdo con el psicólogo y neurocientífico Sergio Bravo, una patología urbana se define como: "una enfermedad física o mental que padece una persona a la hora de habitar la ciudad o una determinada región" (citado en Barquero y Martínez, 2016). Por otro lado, Iglesias Martínez (2012) señala que son "disfunciones que han ido desarrollando los ecosistemas urbanos". Se comprende entonces el concepto de patología urbana como un fenómeno en el que la persona y la ciudad influyen recíprocamente atentando contra su salud. Pero existen al menos, cuatro factores (Guerry y Rivera, s.f.) que determinan una patología urbana:

- Sobrepoblación. - Este fenómeno ha generado que las ciudades y zonas metropolitanas afronten problemas de hacinamiento, escasez de vivienda, inseguridad, congestión vehicular y peatonal.

- Contaminación del aire. - Es producida por la emisión de diversos contaminantes emitidos por vehículos e industrias y son perjudiciales para la salud humana. Se ha establecido una fuerte relación de esta problemática con la afección de la función respiratoria, el flujo del oxígeno en el cuerpo, enfermedades respiratorias cardiovasculares, dermatológicas y con la presencia de diversos tipos de cáncer.

- Contaminación sonora. - Los emisores de ruido más comunes son el transporte, las obras públicas y las industrias. 
- Contaminación visual. - Afecta al sistema nervioso central, ya que cuando una imagen supera el máximo de información que el cerebro puede asimilar (estimado de $4 \mathrm{bit} / \mathrm{seg}$.) se produce un estrés visual, el panorama perceptual se vuelve caótico y la lectura ordenada del paisaje se hace imposible.

Por ello, el estudio de las patologías urbanas deja en manifiesto que la cualidad del entorno influye en la calidad de vida de las personas, por ello los espacios, deberán considerar elementos y estrategias que mitiguen el ruido, mejoren la calidad del aire y propongan visuales naturales agradables a las personas.

Salud urbana

Según Brunelli (2012) la salud implica un bienestar psicofísico integral, en el que intervengan factores como el estilo de vida, el entorno físico y el entorno social. Se establece entonces, que la salud de las personas está ligada al ambiente urbano construido y la naturaleza puede ser un factor clave que contribuya bienestar en los centros urbanos; esta a su vez permitiría mitigar problemas físicos y mentales a través de lo que Brunelli considera "la naturaleza en la ciudad" (2012). Otros autores como Contel \& Llobell (2007) enfatizan también la importancia de proporcionar áreas verdes a la ciudad, que cumplan el rol de ser pulmones urbanos, convirtiendo a las urbes en espacios más sanos. Cabe señalar que la calidad de vida social repercute en la salud; es decir, en la medida en que las relaciones entre las personas mejoren se fortalezcan, se puede alcanzar un grado de equilibrio social - incluyendo los lazos de convivencia, confianza e interacción — que contribuirá con los ideales de salud. Se establece así a la salud urbana como un objetivo que propone mejorar la calidad de vida de las personas, considerando la importancia de la naturaleza, las relaciones sociales y los espacios destinados a la recreación y la actividad física en su entorno urbano. Esto implica una planificación urbana con visión holística, lo que debe ejercer un rol importante como impulsor de decisiones y proyecciones en el marco del desarrollo de las ciudades.

Salud integral y espacios saludables

Por su parte, la OMS define a la salud como "un estado de completo bienestar físico, mental y social y no solo la ausencia de afecciones o enfermedades", determinando de esta manera tres dimensiones del ser humano. La escritora Elena G. White sostiene que la persona posee cualidades físicas, mentales y espirituales, las mismas que han perdido su potencial debido a inadecuados estilos de vida, para ella la salud integral no 
es una mera teoría sino más bien un principio latente en el día a día (Consejos sobre la salud, 143). Se comprende entonces que las dimensiones de una salud integral son: lo físico, mental, social y espiritual. El estudio de estas dimensiones deberá ser un condicionante fundamental al momento de proyectar espacios de bienestar, terapia o prevención de la salud, ya que no considerar uno de estos aspectos significa fragmentar a la persona y poner en riesgo su salud integral.

En otro orden de cosas, existen investigaciones que destacan la importancia del espacio en la salud de la persona, por ejemplo, Ulrich (1984) realizó un estudio entre los pacientes que se sometieron a cirugía de la vesícula biliar, y una notable conclusión fue que los pacientes asignados a una habitación con vista hacia la naturaleza después de su cirugía, requerían menos analgésicos fuertes en comparación con aquellos que fueron asignados a una habitación con vista a una pared de ladrillos, lo que sugiere la importancia de contemplar la naturaleza como un analgésico natural (van Den Berg, 2005). Por ende, las investigaciones demuestran que la presencia de elementos naturales en la recuperación de un paciente puede acelerar su proceso de mejoría, pues la vegetación, por ejemplo, tiene la capacidad de alterar la actividad eléctrica del cerebro, reduciendo el nivel de hormonas que causan estrés y atenúa la tensión muscular (Ortega, 2011).

En conclusión, un espacio saludable es aquel que considera las dimensiones biológica, psicológica, social y espiritual de la persona para mejorar su salud y acelerar el proceso de curación; elementos como el agua, el aire y el sol, pueden cumplir un rol fundamental al momento de diseñar ambientes arquitectónicos. Esto, unido al manejo adecuado de los componentes de la arquitectura como la luz, los colores, el sonido, los olores, los materiales y las proporciones, lograran en el paciente una mejoría significativa.

\section{Relación de los componentes arquitectónicos con el individuo}

A fin de comprender la relación entre la arquitectura y la persona, es necesario comprender lo que se conoce como proceso perceptivo. Sensación y percepción se clasifican como los primeros procesos que acontecen en el hombre para comprender el mundo que lo rodea. La sensación es el modo cómo el hombre recibe la información proveniente del mundo a través de los sentidos; esa información es un dato fragmentado y segmentado de la realidad. Como segundo momento de este proceso aparece la percepción y se configura como el proceso organizador de aquellos datos separados, interpretando de esta manera una información clara, precisa y ordenada (Delclaux y Seoane, 1982). 
En el proceso perceptivo, distintas zonas del cerebro analizan los estímulos de los sentidos, determinando así que la información llegue a ambos hemisferios del córtex cerebral (Bedolla, 2002). Ortega (2011) sugiere que, puesto que la información sensorial permite la estimulación de ambos hemisferios, el espacio arquitectónico puede potenciar las habilidades de cada uno de los hemisferios cerebrales estimulando con ello las dos formas de pensamiento-lógico y creativo. A continuación, se estudian los elementos de la arquitectura y su relación con el individuo.

La luz y el individuo

La luz es un agente físico que hace visibles los objetos (DRAE, 2018). En la arquitectura, la presencia de la luz es importante pues favorece el desarrollo de las formas; sin embargo, su influencia no sólo es óptica, ya que impacta también la fisiología, psicología y el espíritu de las personas. Fisiológicamente, la luz produce una hormona llamada melatonina, que es la responsable del sueño, influye en el sistema inmunológico, en el envejecimiento, el cáncer, enfermedades cardiovasculares y psiquiátricas. Su adecuada segregación es fundamental para el bienestar y esto se consigue siguiendo los ritmos habituales de las variaciones de la luz solar (Salvador, 2010). Por otro lado, el impacto psicológico de la luz según Camacho, Reynoso y Piña (2015) son la liberación de serotonina y endorfinas al organismo, disminuyendo el estrés y fortaleciendo la autoestima. Por último, la luz desde el punto de vista espiritual se identifica con la vida, la sabiduría y la Deidad (Sal 43: 3; 27: 1).

El color y el individuo

Los colores inspirados en la naturaleza, tienen un impacto positivo en la recuperación de pacientes al igual que el uso de materiales y visuales hacia áreas verdes. Por el contrario, ambientes que carecen de referencias a elementos naturales en cuanto al color, materiales, o estímulos visuales, pueden aumentar la ansiedad y causar efectos nocivos (Hill, 2008). De acuerdo a Ortega (2011), los colores pueden generar dinamismo, alegría, sobriedad, tristeza, neutralidad y estimular diversas reacciones psicológicas; simbólicamente propone que, los colores también tienen un mensaje ya sea referido al amor, la fe, la sabiduría, esperanza o la pureza. 
El sonido y el individuo

Hacia finales del siglo XIX, la ciencia comienza a estudiar los efectos físiológicos de las ondas sonoras en la persona (Mendoza, 2018) y se determinó que pueden influir en el funcionamiento cardiaco, la frecuencia respiratoria, la tensión arterial y la función endocrina, además de brindar un efecto calmante y armonizante sobre el organismo. Por otro lado, cuando el sonido supera los límites soportables por el oído humano, se convierte en ruido (DRAE, 2018), la principal consecuencia psicológica del ruido es la sensación de desagrado, molestia y pérdida de concentración. Además, niveles altos de inmisión sonora pueden provocar trastornos en la salud mental como cefaleas (dolor de cabeza), inestabilidad emocional, irritabilidad, agresividad y síntomas de ansiedad (Ortega, 2011).

El olor y el individuo

El olfato humano distingue entre más de 10,000 aromas; los olores a los que se expone la persona, determinan el $70 \%$ de las emociones. Lo que sugiere que, si una persona respira más de 23000 veces al día, tenemos más de 23000 oportunidades diarias para estimular el olfato de una persona (Ortega, 2011). Recientes investigaciones indican que el olor estimula el sistema nervioso central, el cual modifica el estado de ánimo, la memoria, las emociones, el sistema inmunitario y el sistema endocrino (Maldonado, Gutierrez y Lobato, 2012). En un contexto de new age, Zohar y Marshall (2002) hasta declaran que el sentido del olfato estimula energías creativas nuevas, despierta la imaginación y ayuda al crecimiento espiritual y al encuentro con uno mismo.

La vegetación y el individuo

Científicos del Laboratorio de Paisaje y Salud de la Universidad de Illinois en Estados Unidos, realizaron una investigación cuyos resultados concluyen que la naturaleza es un componente esencial para la buena salud y un factor influyente en el comportamiento humano. Así también, de acuerdo a Muñoz (2015), la naturaleza reduce los niveles de citoquinas inflamatorias que están relacionadas con el estrés, la diabetes y la depresión; reduce además la glucosa en la sangre, mejora el sueño, aumenta los niveles de DHEA, que tiene un efecto anti obesidad, anti diabetes y protege el sistema cardiovascular, finalmente, aumenta el número de linfocitos NK que ayudan a combatir el cáncer, las infecciones y enfermedades autoinmunes. Por otro lado, el valor simbólico de la naturaleza está asociado a una conexión con Dios. Pues desde que el hombre fue 
creado, el Jardín del Edén fue su primera casa, y allí Dios se manifestaba, la naturaleza se muestra como una carta de amor del creador. La mente sensible, puesta en contacto con el milagro y el misterio del universo, no puede dejar de reconocer la obra del poder infinito (La educación, 89).

Los materiales

Según ciertos estudios, los materiales tienen propiedades que se clasifican en cinco tipos: físicos, mecánicos, químicos, ecológicos y sensoriales (Baquera, 2016). Las propiedades señoriales se perciben mediante los sentidos y permiten la relación directa entre el usuario y el objeto. Se establecen así cuatro grupos de estímulos sensoriales:

- Sabor - olor

Dulce, picante, solventes.

- Auditivo

Resonante, apagado, ruidoso, hueco, silencio.

- Táctil

Cálido, frío, suave, flexible, rígido, rugoso, áspero.

- Visual

Claro, transparente, opaco, traslúcido, reflectante, brillante, mate, texturizado.

Por ello, son los sentidos los que permiten percibir las formas y los materiales, así también, las sensaciones y emociones que estos transmiten; lo que indica que los materiales pueden poseer características olfativas, táctiles, visuales, auditivas y gustativas (Gómez, 2012).

\section{Conclusiones}

La salud integral de la persona está determinada por cuatro dimensiones: físico, mental, social y espiritual, y se ve condicionada por el entorno que lo rodea, ya que el ambiente tiene la capacidad de contribuir al bienestar o la enfermedad en el individuo. En arquitectura, los componentes que la rigen (luz, color, sonido, olor, vegetación y materiales) tienen también la cualidad de influir en cada una de las dimensiones del ser humano y contribuir con los procesos de mejoría de los pacientes-usuarios en ambientes orientados a la salud. Considerar los aspectos físico, mental, social y espiritual de la persona y su relación con el espacio arquitectónico será fundamental al momento de proyectar una construcción o un ambiente, ya que dejar de lado alguna de éstas condicionantes significaría fragmentar al ser humano. 


\section{Referencias}

Baquera, I. (2016). Exploración de las propiedades sensoriales de materiales no convencionales para su uso en diseño de sillas (Monografía). Universidad Politécnica de Madrid. Madrid, España.

Barquero, M. y Martínez, M. (2016). Arquitectura como terapia emocional: Solución a la depresión (Tesis de licenciatura). Universidad Véritas, San José, Costa Rica.

Brunelli, L. (2012). Urbanismo y Salud Urbana. Andalucia, España: La ciudad viva. Recuperado de http://www.laciudadviva.org/blogs/?p=13144

Camacho, E., Reynoso, L. y Piña, J. (2015). Análisis teórico y experimental en psicología y salud. Guadalajara, México: Iteso.

Contell, B. J. y Llobell, L. A. (2007). El urbanismo Saludable. Revista de salud ambiental, 12 (7), 215-220.

Delclaux, I. y Seoane J. (1982). Psicología cognitiva y procesamiento de la información: teoría, investigación y aplicaciones. Madrid, España: Pirámide.

Gómez, M. (2012). Aulas multisensoriales en educación especial. Vigo, España: Ideaspropias Editorial S.L.

Guerry, Ariel y Rivera, J. (2019). Propuesta de evaluación de impacto ambiental vial para la Ciudad de la Plata. La Plata, Argentina.

Khzam, E. (2008). La percepción ambiental como significación del paisaje: implicancias teóricas desde la relación del ser humano y el entorno. Revista Ambiente Total, 1 (1), 2-8.

Maldonado, O., Gutiérrez, K. y Lobato, C. (2012). El sistema olfatorio: el sentido de los olores. Revista de divulgación científica y tecnológica de la Universidad Veracruzana, 25

Recuperado

de

https://www.uv.mx/cienciahombre/revistae/vol25num2/articulos/sistema/

Mendoza, J. (2018). Ecovisiones: Metaperspectivas y tradiciones.

Ortega, L. (2011). La arquitectura como instrumento de cura (Tesis de licenciatura). Universidad Técnica Particular de Loja. Loja, Ecuador.

Van den Berg, A. E. (2005). Health impacts of healing environments. Groninga, Holanda: UMCG.

White, E. G. (1989). Consejos sobre la salud. Buenos Aires: ACES.

Zohar, D. y Marshall, I. (2002). Inteligencia Espiritual. España: Plaza \& Janes Editories, S.A. 